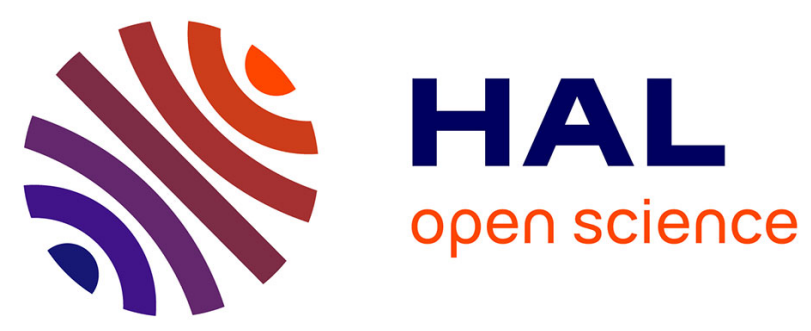

\title{
Effect of black silicon disordered structures distribution on its wideband reduced reflectance
}

\author{
David Abi Saab, Shermila Mostarshedi, Philippe Basset, Stéphane Protat, \\ Dan E. Angelescu, Elodie Richalot
}

\section{- To cite this version:}

David Abi Saab, Shermila Mostarshedi, Philippe Basset, Stéphane Protat, Dan E. Angelescu, et al.. Effect of black silicon disordered structures distribution on its wideband reduced reflectance. Materials Research Express, 2014, 1, pp.45045 - 45045. 10.1088/2053-1591/1/4/045045 . hal-01444437

\author{
HAL Id: hal-01444437 \\ https://hal.science/hal-01444437
}

Submitted on 24 Jan 2017

HAL is a multi-disciplinary open access archive for the deposit and dissemination of scientific research documents, whether they are published or not. The documents may come from teaching and research institutions in France or abroad, or from public or private research centers.
L'archive ouverte pluridisciplinaire HAL, est destinée au dépôt et à la diffusion de documents scientifiques de niveau recherche, publiés ou non, émanant des établissements d'enseignement et de recherche français ou étrangers, des laboratoires publics ou privés. 


\title{
Effect of black silicon disordered structures distribution in its wideband reduced reflectance
}

\author{
D Abi Saab, S Mostarshedi, P Basset, S Protat, \\ D Angelescu and E Richalot \\ Université Paris-Est, 5 boulevard Descartes, Cité Descartes - \\ Champs-sur-Marne, Marne-la-Vallée Cedex 2, 77454, France \\ E-mail: abisaabd@esiee.fr
}

\begin{abstract}
We present a simple and accurate model for the reflectance simulation of black silicon (BSi) based on the finite element method (FEM). Normalized-root-mean-square error (NRMSE) with experimental measurements below $0.25 \%$ has been obtained for wavelength range between 450 and $950 \mathrm{~nm}$. The model is made of a four basic-shape cell whose dimensions are extracted from an accurate topography of the BSi obtained by FIB-SEM tomography. Additional BSi modelling techniques studied, that takes in account the BSi irregular topography, demonstrates an important influence of the local structure height variation in the BSi surface spectral reflectance.
\end{abstract}

Keywords: Nanostructures, reflectivity, measurement, finite element method, silicon, surfaces.

\section{Introduction}

Due to their low reflectance of visible light at a wide range of incident angles, black surfaces consisting of dense structures with sub-wavelength dimensions are promising materials that have been used in applications where light trapping or suppression of stray light are required. Although black surfaces are usually referred to low reflectance in visible light, these materials can also have very low reflectance in a wider spectral range including near infrared wavelengths.

Several surface treatment techniques on crystalline silicon have led to near zero reflectance surfaces in a broad wavelength and angles of incidence range, consisting of periodic arranged silicon nanopillars [1], nanowires [2] or disordered distributed silicon needle-like structures also called black silicon (BSi) [3]. Several techniques such as cryogenic reactive ion etching (cryo-RIE) [4], Deep RIE Bosch process [5], nano-particle assisted wet etching [6] and laser ablation [7] are currently employed to fabricate BSi. While many of these techniques consist of a mask-less process and do not dependent of the Silicon crystal orientation, one advantage of cryo-RIE fabrication 
method is that by tuning the reactor parameters, BSi surfaces can be obtained with a great variety of structures dimensions and shapes [8].

The importance of the improvement of these low reflectance micro and nanostructured silicon surfaces lies in the prospect of increasing the efficiency of photovoltaic cell designs due to their light trapping effects, as already demonstrated by devices of improved efficiency [9]. Additionally, other applications related to BSi optical properties includes the responsivity improvement of silicon based infrared photo-diodes [10] due to greater absorption in near infrared wavelength range and the use of BSi to enhance thermal absorption in energy harvesters [11].

Numerical simulations of $\mathrm{BSi}$ based structures are useful in order to find the relation between the BSi topography and its reflectance spectrum. Matching between simulated reflectance and measurements of BSi structures or similar ones has been demonstrated to some extend in terms of accuracy using different methods such as rigorous coupled wave analysis (RCWA) [12], finite-difference time-domain (FDTD) [13] and finite element method (FEM) [11]. Simulations can be performed by means of modelling an equivalent unit cell structure with bi-periodic boundaries as illustrated in Figure 1 which may consist of a basic shape (e.g. cone, cylinder) [11, 12] or real surface topography based on a 3D model reconstruction [13, 14].
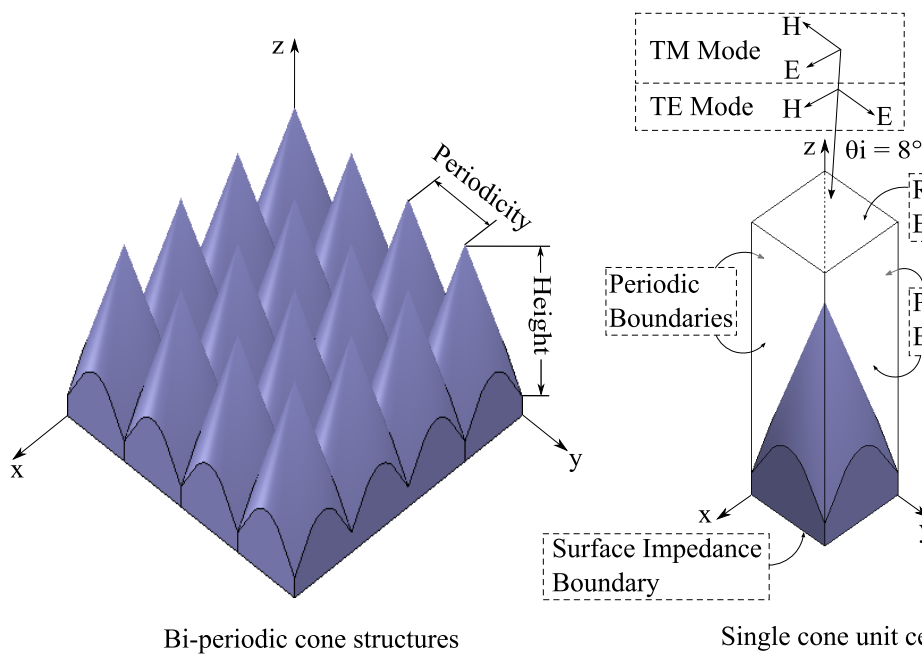

Boundary

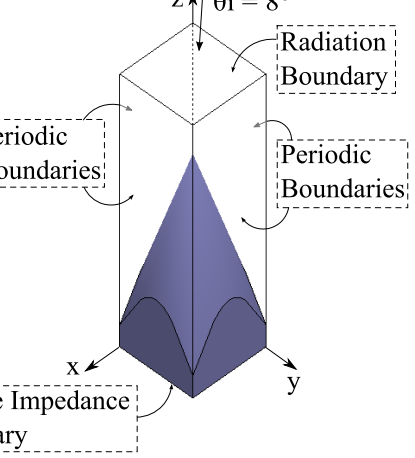

Single cone unit cell

Figure 1. View of a large bi-periodic micro-structured surface from which a unit cell is defined with periodic boundaries for numerical simulation of reflectance.

Using FEM numerical simulations, parametric studies of structures with dimensions based on side view black silicon SEM micrographs have shown similar tendencies as the ones obtained experimentally [11]. However, accuracy of such approach is strongly affected due to the difficulty of modelling real BSi surfaces with their wide range of rugosity dimensions and their disordered distribution. In this 
case the definition of the equivalent unit cell is not obvious, the key point lying in the choice of the appropriate bi-periodic unit cell that includes the influence of the heterogeneity of BSi structuring. In this paper several methods of modelling BSi unit cells are proposed where reflectance simulations provide improved accuracy in regard to their agreement with the ones obtained by measurements.

In the following section, a statistical analysis is performed on accurate $3 \mathrm{D}$ model representations of two BSi samples obtained by FIB-SEM tomography [15], with slightly different geometries. Once the geometric properties of the $\mathrm{BSi}$ samples are determined, three methods of modelling the bi-periodic unit cell are presented in the third section. Their reflectances obtained by numerical simulations are compared with measurements carried out by means of an integrating sphere.

The first method consists of modelling small regions of the actual BSi surface topographies obtained from the three-dimensional reconstruction. Periodic boundary conditions are imposed on the side faces of the small region in order to obtain an infinite surface avoiding finite surface edge effects. The simulation based on actual BSi topography is however restricted to very small regions due to the required computational effort, consequently this approach cannot handle the surface edging heterogeneity.

The second method incorporates a single equivalent protuberance as unit cell that has a similar height distribution as the one obtained from a large BSi surface; the better choice of the equivalent shape will be discussed.

The third method comprises an equivalent structure made of several elementary shapes having different dimensions to include the effect of the disordered distribution of the BSi structures.

\section{Black Silicon geometric properties}

\subsection{BSi fabrication process and $3 D$ model reconstruction}

Two Czochralski-grown, p-doped, $<100>$ oriented, silicon wafers of thickness $525 \mu \mathrm{m}$ and bulk resistivity of 1-20 $\Omega . \mathrm{cm}$, labelled S01 and S02, are processed with different cryo-RIE parameters (Table 1) in order to produce BSi with different geometrical properties. A tilted view SEM micrograph of BSi samples S01 and S02 are shown in Figures $2 \mathrm{a}$ and $2 \mathrm{~b}$.

The BSi structures obtained by cryo-RIE consist of disordered distribution of pores or cylindrical holes of sub-micrometric dimensions. The intersection of these pores results in some needle-like dense sub-wavelength structures with high aspect ratio that produce a diffuse scattering effect on incident light. The reflectance of BSi surfaces is drastically reduced compared to Silicon planar surfaces in a wide wavelength range including visible light and near infrared and for a wide range of incident angles 
Table 1. BSi cryo-RIE fabrication parameters

\begin{tabular}{|c|c|c|}
\hline cryo-RIE Parameters & Sample S01 & Sample S02 \\
\hline Temperature $\left({ }^{\circ} \mathrm{C}\right)$ & -110 & -120 \\
ICP Power $(\mathrm{W})$ & 1000 & 1000 \\
Bias Voltage (V) & -20 & -10 \\
$\mathrm{O}_{2}$ flow (sccm) & 20 & 10 \\
$\mathrm{SF}_{6}$ flow (sccm) & 200 & 200 \\
Pressure $(\mathrm{Pa})$ & 1.6 & 1.5 \\
Processing time (min.) & 15 & 10 \\
\hline
\end{tabular}

(as discussed later in Section 3.1).

Followed the BSi fabrication, we proceed to reconstruct a three-dimensional model of the BSi samples. 3D reconstructions based on SEM imaging can provide partial information on the surface topography [14], but due to the occlusion caused by the very dense high aspect ratio structures a 3D reconstruction method based on FIBSEM is used instead [15], with precision in the order of $10 \mathrm{~nm}$ along $x$ and $y$ axes (10 $\mathrm{x} 10 \mu \mathrm{m}$ sample size), and approx. $100 \mathrm{~nm}$ along $z$ axis (see Figure 1 for the axes).

Figures $2 \mathrm{c}$ and $2 \mathrm{~d}$ show the SEM tilt view of a single structure from BSi sample S01 compared to the 3D model reconstruction obtained by FIB-SEM tomography.

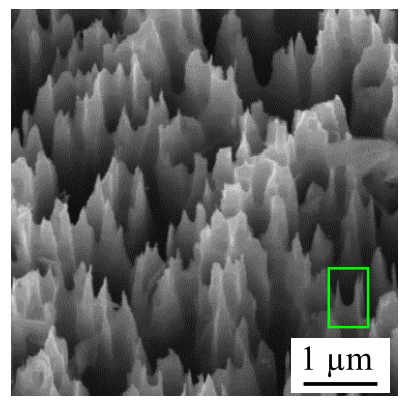

(a)

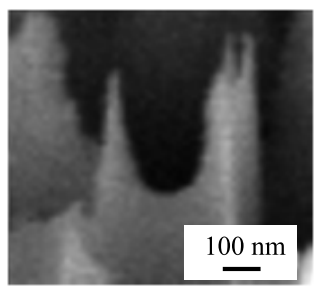

(c)

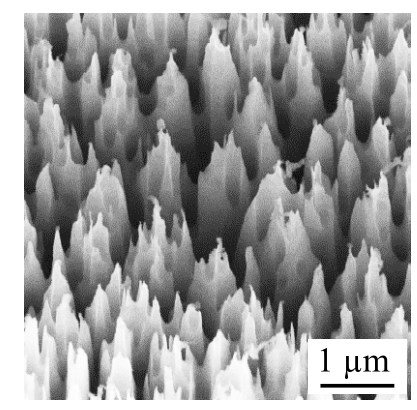

(b)

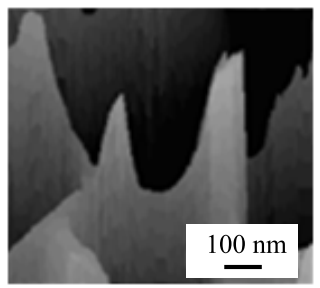

(d)

Figure 2. SEM micrograph (20 tilt view) of BSi samples S01 (a) and S02 (b). A zoomed area of the SEM corresponding to BSi sample S01 (c) is compared with the $3 \mathrm{D}$ model reconstruction of the same area of the sample (d). 


\subsection{Statistical analysis of BSi samples}

By analysing the 3D model of the BSi samples, the height and periodicity distributions are determined. The height distribution is obtained from all the data points of the 3D model. The statistical values such as the mean $h_{\mu}$ and standard deviation $h_{\sigma}$ of the surface height are calculated.

Figure 3a shows the height distributions for BSi samples S01 and S02. Although both samples have similar distributions, the mean structure height of BSi sample S01 is higher than $\mathrm{S} 02$. In addition, the larger $h_{\sigma}$ value of BSi sample S01 suggests a greater variation of structures height and therefore more inhomogeneity. The local variations of structure height can be observed in the cross-section view of BSi sample S02 (Figure 3b) with the difference between global and local height levels and the variation of the distance between the peaks and the holes along the surface.

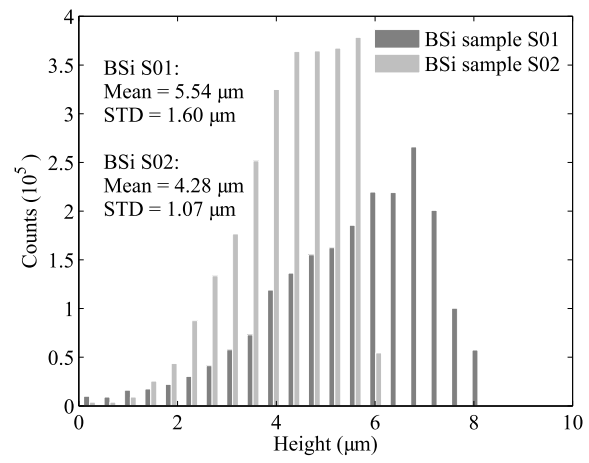

(a)

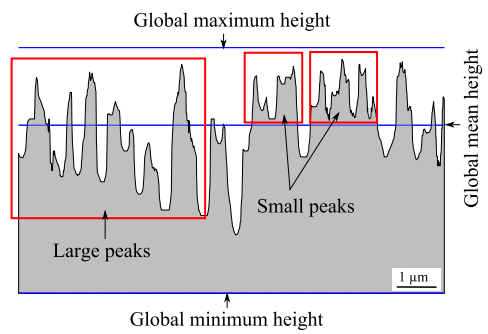

(b)

Figure 3. Height distributions of BSi samples S01 and S02 (a), indicating their corresponding mean and standard deviation values. (b) BSi sample S02 threedimensional model cross-section view with lines describing maximum, minimum and mean global heights. The small peaks observed on top of larger scale structures justify the left-skewed height distribution (the distribution mass tends towards the maximum global height).

The periodicity distributions of the BSi samples shown in Figure 4a indicates a larger mean periodicity $P_{\mu}$ of BSi sample S01 than S02. The distributions are obtained 
by measuring the distances between the centre of each hole observed in the top view of the 3D model and the centre of its closest neighbour holes, as illustrated with segments in Figure 4b.

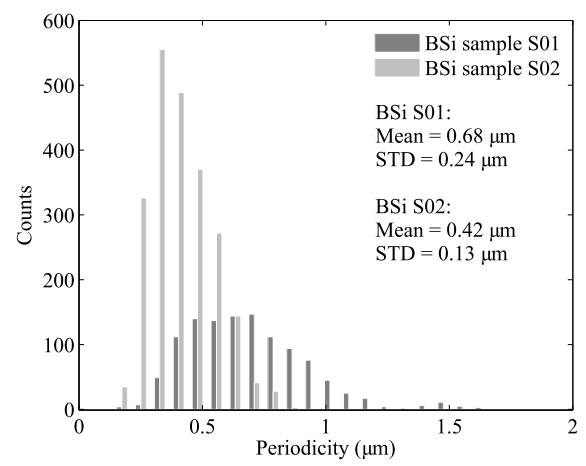

(a)

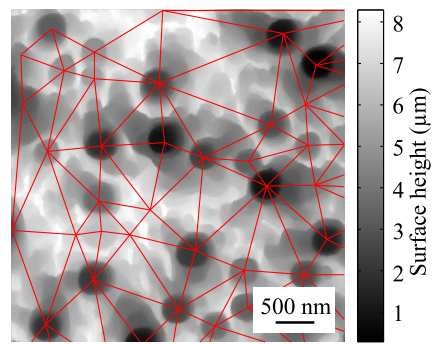

(b)

Figure 4. Periodicity distribution of $\mathrm{BSi}$ samples $\mathrm{S} 01$ and $\mathrm{S} 02$ (a) and small section top-view of BSi sample S01 three-dimensional model (b). Red line segments represent the measured distances between neighbour holes and greylevel scale shows the height relative to the global BSi sample height.

The mean surface normal angle $\alpha_{N}$ defined as the angle between the normal relative to local surface and the substrate horizontal back surface is also calculated in both samples, where values of $79.44^{\circ}$ and $78.49^{\circ}$ are obtained for BSi samples S01 and S02 respectively.

\section{Black Silicon optical properties}

\subsection{BSi reflectance measurements}

Reflectance measurements are performed on the two BSi samples S01 and S02. The setup consists of an integrating sphere, a halogen lamp light source and a spectrometer. The sketch of the measurement setup is shown in Figure 5. The reflectance $R$ being determined as the ratio of the total reflected radiant flux to that of a reference 
diffuser [16], a certified $2 \%$ diffuse reference is used. The light beam enters the integrating sphere with an incident angle of $8^{\circ}$ from normal and the reflected flux is collected hemispherically. The evaluated wavelength range of the reflectance measurements is between $450 \mathrm{~nm}$ and $950 \mathrm{~nm}$, the area of the sample being measured is approximately $28 \mathrm{~mm}^{2}$. Three spectra measurements are necessary to calculate the sample reflectance $R_{B S i}$ : the dark spectrum $I_{d}$ where the light source is blocked, the reference spectrum $I_{\text {ref }}$ and the sample spectrum $I_{B S i}$. Considering the null dark reflectance $R_{d}$ and the known reference reflectance $R_{r e f}$, the sample reflectance is obtained by a linear interpolation, as shown in Equation 1:

$$
R_{B S i}=R_{d}+\frac{\left[I_{B S i}-I_{d}\right] *\left[R_{r e f}-R_{d}\right]}{\left[I_{r e f}-I_{d}\right]}
$$

Figure 6 shows the total, specular and diffused reflectances of BSi samples S01 and S02. By changing the integrating sphere configuration, total and diffused reflectances for both samples are measured. The specular component is calculated by subtracting the diffused component from total reflectance.

We first of all notice that the diffused component, due to the surface rugosity, is preponderant. Therefore, reflectance simulations methods based on multilayered media combined with effective medium theory techniques $[17,18]$ are not suitable for these surfaces. The specular component is mostly constant with the wavelength whereas the diffused one increases with the wavelength. Although both samples have different structure dimensions, they present similar mean aspect ratios leading to very close reflectance behaviours, the total reflectance of S02 being slightly lower (mean reflectance of $1.45 \%$ against $1.55 \%$ for S01).

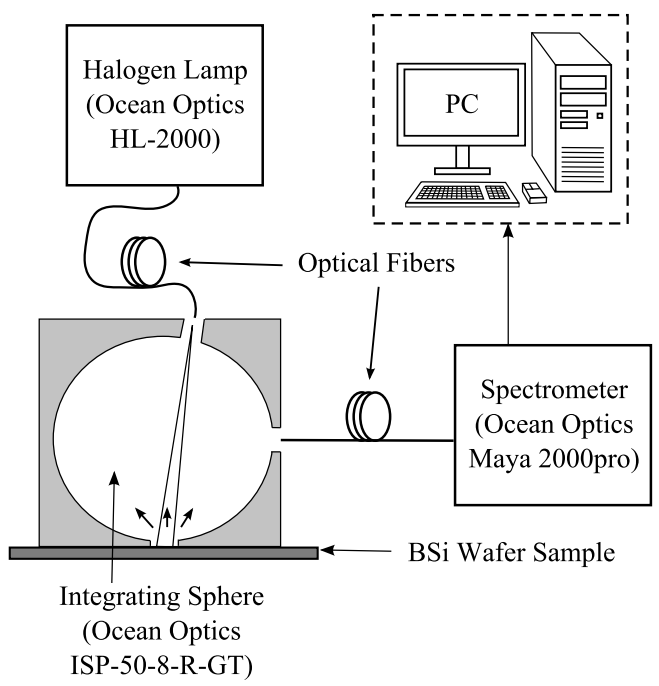

Figure 5. BSi sample reflectance measurement setup. 


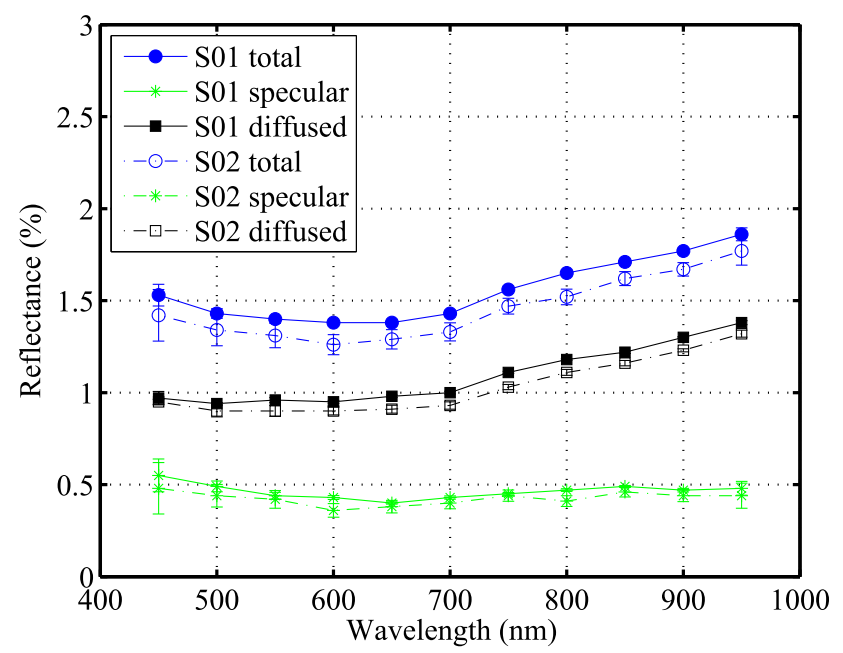

Figure 6. Reflectance measurements of BSi samples S01 and S02. Error bars represent the standard error calculated from 4 measurements on different regions of the sample.

\subsection{Numerical reflectance simulation methodology}

Reflectance simulations in the visible light wavelength range are performed using ANSYS HFSS software. The bulk silicon material permittivity and tangent loss in function of wavelength required for the simulations are based on information obtained from the literature [19].

Periodic boundaries are imposed in the side faces of the unit cell. The source consists in a plane wave with an incidence angle of $8^{\circ}$ with respect to the wafer back surface normal vector (Figure 1). At the bottom of the simulated volume, an impedance condition is used to simulate the thickness of the silicon substrate of $500 \mu \mathrm{m}$ and a perfect conductor is placed under the silicon layer to further match the conditions of the reflectance measurement setup.

The reflectance spectrum is evaluated at the top face of the cuboid denoted in Figure 1 as the radiation boundary. As non-polarized light is used in measurement, the reflection coefficient $S_{11}$ as a function of wavelength is evaluated for both TE and TM polarizations, the related reflectances being calculated according to Equation 2. The total reflectance $R$ is obtained from the average of both polarizations (Equation 3). The reflectance simulations include both specular and diffused components, since the $S_{11}$ parameter is calculated using the total scattered electromagnetic fields.

$$
\begin{aligned}
& R_{T E \mid T M}=\left|S_{11}\right|_{T E \mid T M}^{2} \\
& R=\frac{R_{T E}+R_{T M}}{2}
\end{aligned}
$$




\subsection{Methods of modelling the BSi}

\subsubsection{Simulation of real surface topography}

The surface data obtained from BSi reconstructions with FIB-SEM tomography (Section 2.1) can be exported in various three-dimensional digital formats for further analysis. In this section, small regions of the BSi surface topography are imported in HFSS to perform reflectance simulations. The regions are chosen with different mean and STD values of height to observe the influence of different topographic parameters in the reflectance response.

Figure 7 a shows the top view of the reconstructed surface of BSi sample S01. The rectangles represent the regions of this sample which are introduced in HFSS for simulation. Each region has lateral dimensions equivalent to the mean periodicity (estimated in Section 2.2) in the $x$-axis and half of the mean periodicity in the $y$-axis. The final model introduced in HFSS has symmetry conditions applied on boundary surfaces parallel to the incident plane $(z y)$ and periodic conditions applied on the $z x$ plane boundaries. Having a cell with the same lateral boundaries in the $z x$ planes, allows us to perform simulations with off-normal angles of incidence. Figure $7 \mathrm{~b}$ shows the three-dimensional view of region No. 1 unit cell extracted from BSi sample S01. We note that the extracted region consists of a half structure hole; once the symmetry boundary condition is taken in account, the unit cell comprises an entire hole.

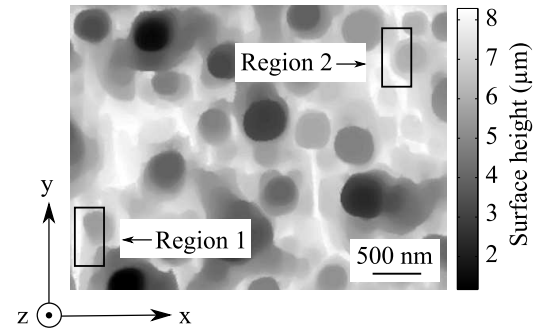

(a)

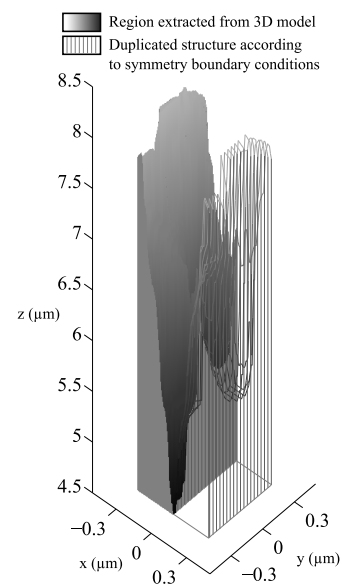

(b)

Figure 7. Top-view of a small part of BSi sample S01 model with rectangles delimiting the extracted regions used as periodic unit cell, the grey-level scale represents the global BSi sample height (a). Three-dimensional view of region No. 1 unit cell extracted from BSi sample S01, comprising the simulated half unit cell and the duplicated half due to symmetry boundary conditions (b).

Table 2 shows the statistical parameters of each region and the global BSi sample 
parameters. The minimum, maximum, mean and STD heights $\left(h_{\min }, h_{\max }, h_{\mu}\right.$ and $h_{\sigma}$ respectively) of each region are relative to their corresponding global BSi sample topographical parameters. The mean depth $d_{\mu}$ is deduced from $h_{\max }$ minus $h_{\mu}$. The mean periodicity $P_{\mu}$ of each region corresponds to the unit cell size being the same as $P_{\mu}$ for the global BSi sample S02 but slightly smaller for BSi sample S01, due to the maximum cell volume imposed by the limited computational resources. We also calculate the mean surface normal angle $\alpha_{N}$ for each region and the height based on the mean surface normal angle $\left(h_{N}=\tan \left(\alpha_{N}\right) * P_{\mu} / 2\right)$.

Table 2. Comparison between the topographic parameters of each region with their corresponding global sample parameters.

\begin{tabular}{|c|ccc|}
\hline \multirow{2}{*}{ Parameters } & \multicolumn{3}{|c|}{ BSi sample S01 } \\
\cline { 2 - 4 } & Total & Region 1 & Region 2 \\
\hline$h_{\min }(\mu \mathrm{m})$ & 0.00 & 4.71 & 6.01 \\
$h_{\max }(\mu \mathrm{m})$ & 8.28 & 8.28 & 8.28 \\
$h_{\mu}(\mu \mathrm{m})$ & 5.54 & 6.67 & 7.07 \\
$d_{\mu}(\mu \mathrm{m})$ & 2.74 & 1.61 & 1.22 \\
$h_{\sigma}(\mu \mathrm{m})$ & 1.60 & 0.98 & 0.70 \\
$\alpha_{N}\left({ }^{\circ}\right)$ & 79.44 & 77.82 & 75.38 \\
$h_{N}(\mu \mathrm{m})$ & 1.83 & 1.58 & 1.31 \\
$P_{\mu}(\mu \mathrm{m})$ & 0.683 & 0.600 & 0.600 \\
\cline { 2 - 4 } & \multicolumn{3}{|c}{ BSi sample S02 } \\
\cline { 2 - 4 }$h_{\min }(\mu \mathrm{m})$ & 0.00 & Region 1 & Region 2 \\
\cline { 2 - 4 }$h_{\max }(\mu \mathrm{m})$ & 6.24 & 5.64 & 4.41 \\
$h_{\mu}(\mu \mathrm{m})$ & 4.28 & 4.18 & 5.21 \\
$d_{\mu}(\mu \mathrm{m})$ & 1.96 & 1.45 & 0.57 \\
$h_{\sigma}(\mu \mathrm{m})$ & 1.07 & 0.84 & 0.30 \\
$\alpha_{N}\left({ }^{\circ}\right)$ & 78.49 & 76.82 & 63.67 \\
$h_{N}(\mu \mathrm{m})$ & 1.02 & 0.89 & 0.42 \\
$P_{\mu}(\mu \mathrm{m})$ & 0.416 & 0.416 & 0.416 \\
\hline
\end{tabular}

In the simulations, the reflectance is evaluated for a wavelength range between $450 \mathrm{~nm}$ and $950 \mathrm{~nm}$ using Equations 2 and 3. Figure 8 shows the reflectance simulation of each region compared with the corresponding BSi sample reflectance measurement.

First, we notice the differences between the simulated reflectance of the regions in the same BSi sample that reflect the influence of the structure heterogeneity in the overall response of a larger surface composed by structures with different dimensions and shapes. In addition, we observe for both samples, the decrease of the average reflectance response with the increase of each region STD height $h_{\sigma}$. In agreement with previous published results [11], the average reflectance is decreased, when the region's heights present a large dispersion.

Additional differences between simulations and measurements such as the presence of peaks in the simulated reflectance curves, the tendency of the curves and the agreement of the reflectances observed only for small wavelength ranges show that 


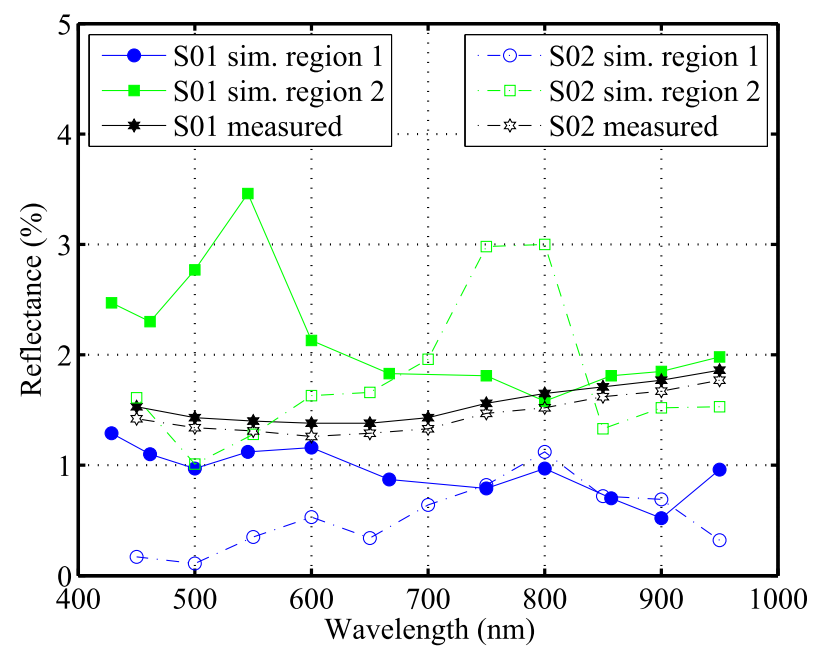

Figure 8. Reflectance simulation of small regions based on real topographic data of BSi sample S01 and BSi sample S02 compared with their respective reflectance measurements

the size of the regions (equal to the mean periodicity) is not large enough to represent the behaviour of the entire $\mathrm{BSi}$ sample. If the size of the evaluated region were incremented then the local geometrical properties would approach the global ones and therefore the reflectance would better match. However, since larger samples cannot be evaluated with FEM due to large memory requirements, an alternative solution is to model an equivalent unit cell structure based on the statistical parameters of the entire BSi sample. In the following sections, two different methods to obtain equivalent unit cell structures are discussed.

\subsubsection{Simulation of a single-structure cell model}

In earlier studies, conic geometrical shapes were used as a unit cell to represent the BSi sample [11], where the dimensions were determined by side view SEM micrographs of the BSi sample. The disadvantage of this method is that one side view SEM micrograph only captures a very small percentage of the sample being studied; therefore, reflectance simulations performed with unit cell structures based on dimensions taken from these images do not provide a good representation of the reflectance of the entire BSi surface.

To overcome this limitation, a two-step approach is proposed. The first step consists of determining a basic shape for the equivalent surface that provides a similar height distribution as the one obtained from the three-dimensional reconstruction of BSi surface. Once the shape is selected, in the second step its dimensions are 
determined based on the information obtained from the statistical values of height and periodicity of its corresponding BSi sample.

In order to find the best equivalent shape for the BSi samples being studied, the height distributions of different geometrical shapes are compared to the BSi samples. Figure 9a shows the comparison of the height distribution between a cone, an inverted cone and the BSi sample S01, with normalized heights.

Since the unit cell is inserted in a bi-periodic squared structure, the cone and inverted cone structures are truncated in a cuboid, to allow the assignment of periodic boundaries on vertical surfaces as shown in Figures 9b and 9c.

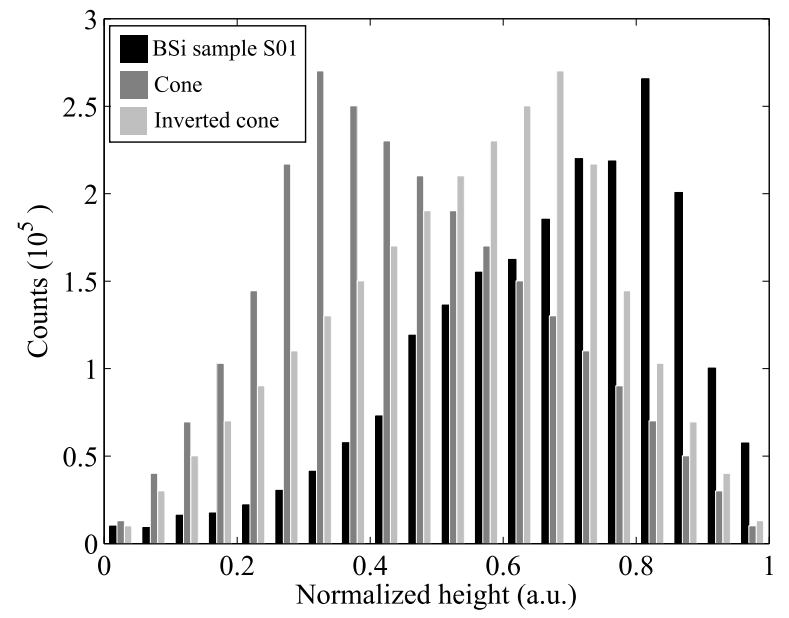

(a)

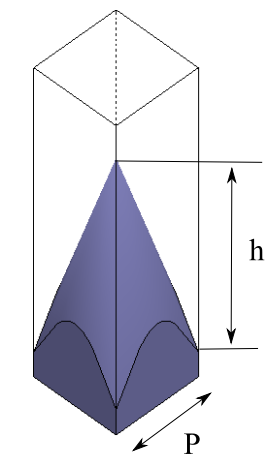

(b)

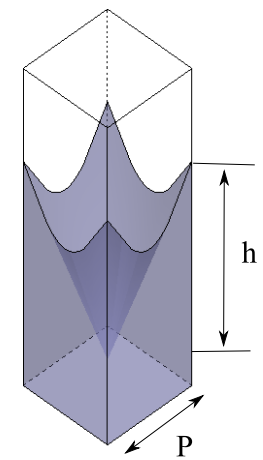

(c)

Figure 9. Comparison of normalized height distribution of a cone, an inverted cone and the BSi sample $\mathrm{S} 01$ (a); 3D view of the cone structure (b) and the inverted cone structure (c)

From Figure 9a, we find that the inverted conic structure height distribution is more similar to the global BSi height distribution than the conic structure. The same 
result is obtained with BSi sample S02.

Using the inverted cone as the equivalent structure shape, we proceed to determine its dimensions based on the global BSi topography data. Three different heights are defined for the equivalent structure: the first one is based on the global mean depth of the structure $d_{\mu}$, the second height $h_{N}$ is based on the mean surface normal angle $\alpha_{N}$ and the third height corresponds to the standard deviation (STD) of the total BSi height distribution $h_{\sigma}$. The reasoning behind the use of the STD lies in the strong variation of the local mean height along the surface observed in Section 2.2. By using only the global mean depth, the effects caused by the small height variations above and below the mean value are not taken in account in the simulation. The width of the unit cell structure is defined based on the mean periodicity of the total BSi sample (Table 2).

Having determined the structure dimensions, the simulations of the equivalent structures of BSi samples S01 and S02 are performed using HFSS to determine the reflectance. The results are then compared with their respective BSi reflectance measurement (Figure 10).

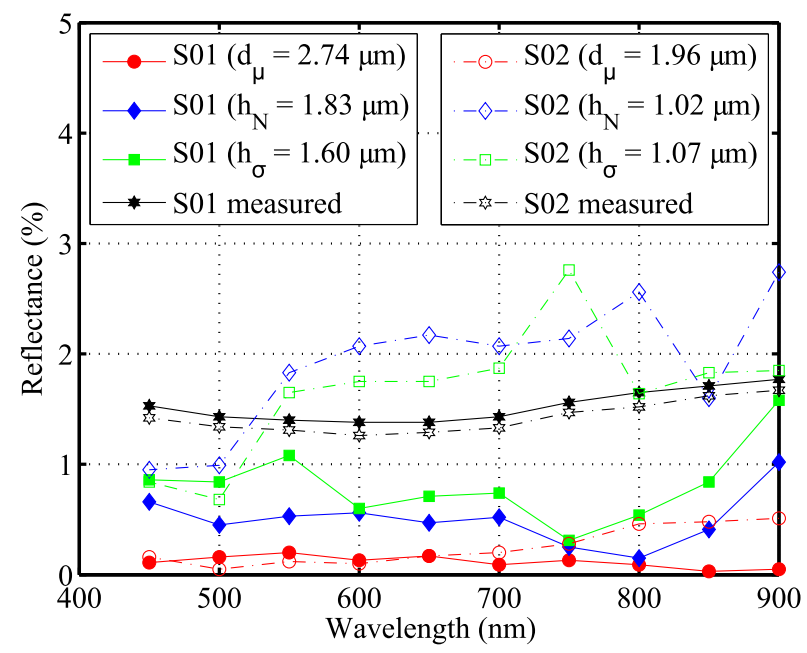

Figure 10. Reflectance simulations of equivalent structure cells based on statistical data from BSi samples S01 and S02 compared with measurements.

In Figure 10 we observe a poor agreement between the reflectance of the single inverted cone structure with height based on the global mean depth $d_{\mu}$ and the reflectance measurements; however, the structures with heights based on the mean surface normal angle $h_{N}$ and global STD of height $h_{\sigma}$, both provide better agreement with the reflectance measurements in both BSi samples. Among these results, the BSi equivalent structure with the height based on the global height STD is the one that 
gives best match over the studied wavelength range. Simulations were also performed using conic structures with the same heights as the inverted cones, but as expected due to the differences in the height distribution of conic structures and BSi samples, none of the simulated reflectances did correspond to the BSi samples measurements.

Although the tendency of the simulated curves obtained with inverted cones agrees with their corresponding measurements, some discrepancies or fluctuations are still observed at certain wavelengths. These discrepancies reflect the remaining difference of the height distribution between the equivalent shape and the BSi sample. In addition, the interferences caused by the perfectly periodic single structure cells result in a reflectance that is more sensitive to frequency variation, whereas interferences in non-periodic BSi samples are expected to be less strong.

In order to overcome these discrepancies, in the next Section an equivalent unit cell consisting of multiple structures is presented, where variations of the inverted cone height and diameter are introduced in order to better match the BSi non-periodic nature.

\subsubsection{Simulation of a multiple-structure cell model}

To include the disordered distribution characteristic of BSi samples in simulations, an equivalent multi-structure unit cell is modelled consisting of 4 inverted cones with variable dimensions based on large scale standard deviations of the periodicity distribution calculated from the total BSi sample. Figure 11 illustrates the arrangement of four individual inverted cones in one multi-structure unit cell.

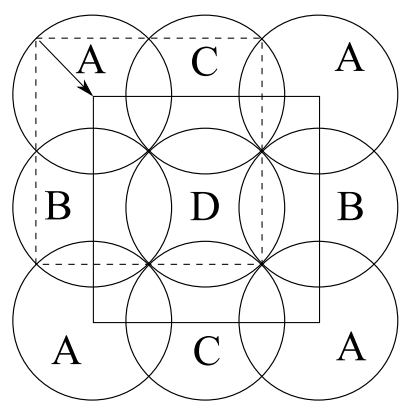

(a)

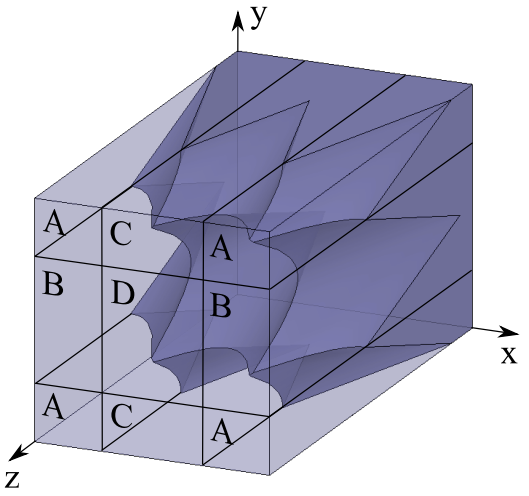

(b)

Figure 11. Representation of multi-structure cell consisting of 4 inverted cones with different dimensions: (a) Top view of the 4 cones structures where the unit cell (represented by the dashed square) is displaced by a half of the periodicity in order to have a symmetrical structure independently of the cone dimensions. (b) $3 \mathrm{D}$ view of the unit cell structure modelled in HFSS

Variation in height and diameter in the multi-structure cell are introduced while 
maintaining the same height mean and height STD of the single structure unit cell defined in the previous section. Based on the mathematical definitions of the statistical mean and STD, we proceed to calculate the height and diameter of each structure for two different multi-structure unit cell configurations. The results of these calculations are presented in Table 3. Only BSi sample S02, presenting the lower reflectance, is considered in this section.

The large scale periodicity standard deviation $P_{\sigma}$ is calculated by dividing the total BSi sample in regions of three times the value of the global mean periodicity. The mean periodicity of each region is calculated followed by the STD between them (38.22 $\mathrm{nm}$ being the large scale STD for BSi sample S02).

Table 3. Individual structure dimensions calculations for the two different configurations of multi-structure unit cell

\begin{tabular}{|c|c|}
\hline $\mathbf{1}^{\text {st. }}$ case & $\mathbf{2}^{\text {nd. }}$ case \\
\hline$A R=h_{\sigma} / P_{\mu}$ & $A R=h_{\sigma} / P_{\mu}$ \\
$d_{A}=P_{\mu}$ & $d_{A}=P_{\mu}$ \\
$d_{B}=P_{\mu}+\sqrt{2} * P_{\sigma}$ & $d_{B}=P_{\mu}-6 / \sqrt{14} * P_{\sigma}$ \\
$d_{C}=P_{\mu}-\sqrt{2} * P_{\sigma}$ & $d_{C}=P_{\mu}+2 / \sqrt{14} * P_{\sigma}$ \\
$d_{D}=P_{\mu}$ & $d_{D}=P_{\mu}+4 / \sqrt{14} * P_{\sigma}$ \\
$h_{A}=A R * d_{A}$ & $h_{A}=A R * d_{A}$ \\
$h_{B}=A R * d_{B}$ & $h_{B}=A R * d_{B}$ \\
$h_{C}=A R * d_{C}$ & $h_{C}=A R * d_{C}$ \\
$h_{D}=A R * d_{D}$ & $h_{D}=A R * d_{D}$ \\
\hline Where: \\
$h \sigma=1.07 \mu \mathrm{m}$ (Standard deviation of height) \\
$P \mu=0.42 \mu \mathrm{m}$ (Mean periodicity) \\
$P \sigma=38.22 \mathrm{~nm}$ (Large scale periodicity STD) \\
\hline
\end{tabular}

The diameter $d$ of each inverted cone is calculated according to the relation defined by the multi-structure configuration; since the inverted cone aspect ratio $A R$ is maintained fixed, the height of the structure can be deduced from the aspect ratio and the base diameter. The calculations based on the mathematical definition of mean and STD do not take into account the overlapping between structures in a squared multi-structure unit cell, therefore the dimensions are manually adjusted to ensure a perfect match of the mean and standard deviation of heights respect to the single structure cell.

Figure 12 shows the heights distributions of the two multi-structure unit cells configurations compared to the single structure unit cell and the BSi sample S02. We observe that due to the calculations based on the definition of mean and STD, we are able to produce different unit cell configurations with almost the same distribution as the single structure unit cell. Following the multi-structure unit cell design, we introduce them in HFSS to simulate their reflectance.

Figure 13 and numerical results in Table 4, compare the simulated reflectance of the multi-structure unit cells with the one obtained from the single structured unit cell and the reflectance measurements. We observe that introducing some height disorder 


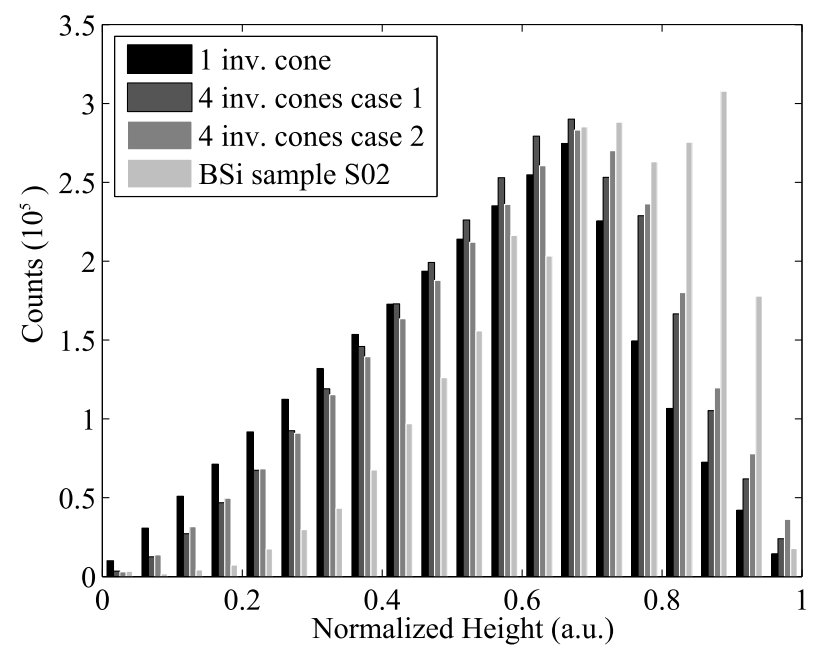

Figure 12. Comparison of normalized heights distributions of the single and multi-structure inverted cone unit cells with the height distribution of the BSi sample S02.

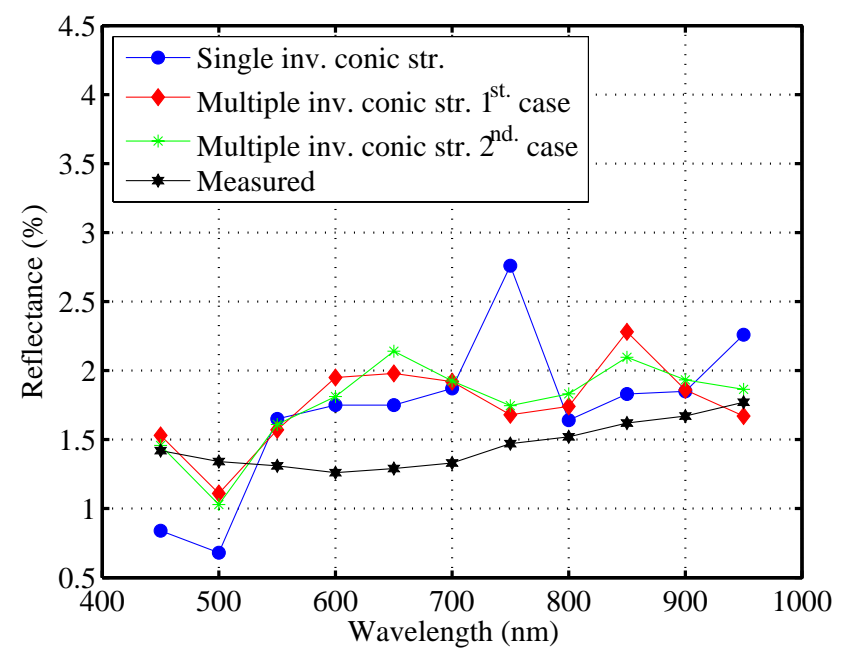

Figure 13. Comparison of reflectance simulation with single and multiple equivalent structures with their respective measured reflectance based on dimensions obtained from BSi sample S02

while maintaining the same topographic parameters of the single structure unit cell, maintains almost the same mean reflectance in the multi-structure unit cells with respect to the single structured unit cell. However, smoother reflectance responses are observed in the multi-structure unit cells that approach the tendency of the BSi measurement. 
This improvement is quantified in Table 4 with NRMSE and STD values. Despite the tendency improvement with respect to measurements, small fluctuations are still observed which could be removed by incrementing the amount of disorder or using a more complex model. Moreover, using the 8 degrees of freedom provided by this model, multi-structure unit cells can be designed with a goal on their height distributions outcome to be similar to the real BSi samples.

Table 4. Mean, standard deviation of reflectance simulations with single and multiple equivalent structures and reflectance measured of BSi sample S02. Normalized-root-mean-square error (NRMSE, normalized to the mean) between reflectance simulations and measurements for wavelength range between 450 and $950 \mathrm{~nm}$.

\begin{tabular}{|c|c|c|c|}
\hline & Mean R\% & STD R\% & NRMSE (\%) \\
\hline Single structure & 1.72 & 0.57 & 0.33 \\
Mult. structure \#1 & 1.75 & 0.30 & 0.24 \\
Mult. structure \#2 & 1.77 & 0.31 & 0.24 \\
Measured & 1.45 & 0.17 & - \\
\hline
\end{tabular}

\subsection{Influence of the source angle of incidence}

An additional interesting aspect of BSi surfaces is their ability to reduce the reflectance independently to the incidence angle of the source light. This property is useful in solar cells applications where maximum amount of light is required independently of the position of the panel with respect to the sun.

Simulations at different angles of incidence (AOI) are performed in the two different configurations of the multi-structure cells presented in Section 3.3.3 using a planar wave excitation at $700 \mathrm{~nm}$ of wavelength, and they are compared to the single inverted conic structure cell. We demonstrate in Figure 14 that very low reflectance response is maintained at different angles of incidence up to $40^{\circ}$, followed by a slightly reflectance increase at greater AOI values for both single and multi-structure cells. In addition by introducing the disorder in the two configurations of the multi-structure cells, a lower reflectance is obtained for intermediate angles of incidence (between $30^{\circ}$ and $60^{\circ}$ ). These results suggest that for surfaces irradiated in different AOI the total hemispherical reflectance of disordered structure distribution (typical in BSi) is reduced compared to strictly periodic structured surfaces of similar dimensions.

\section{Conclusions}

We have presented three modelling techniques of BSi samples to improve the simulated reflectance with respect to the one obtained by measurements. All techniques are based on statistical information of $\mathrm{BSi} 3 \mathrm{D}$ model reconstructions obtained by FIB-SEM 


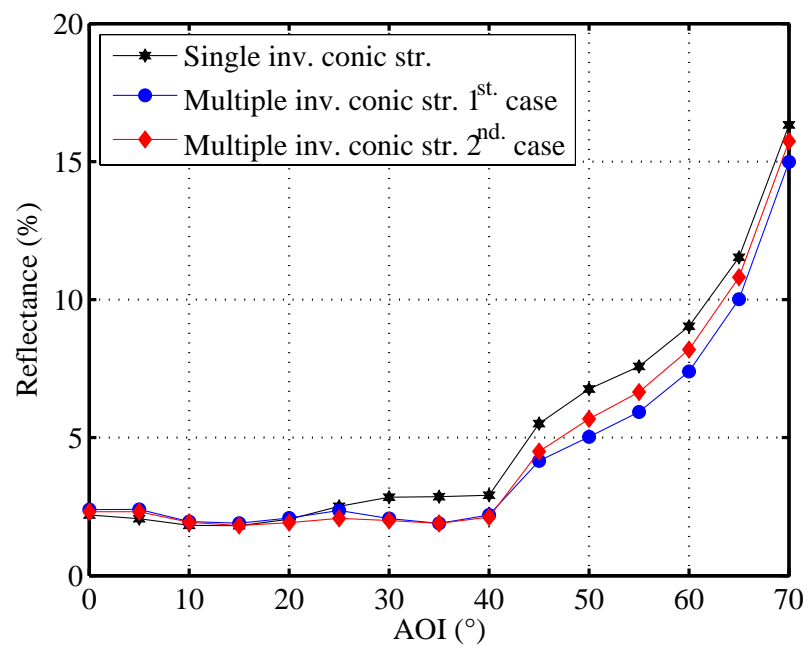

Figure 14. Comparison of reflectance simulations with single and multiple structured unit cells in function of angle of incidence

tomography due to the dense agglomeration of high aspect ratio structures typical in BSi samples. The first technique, consisting of numerical simulations of unit cell structures based on regions of actual BSi topography, provides partial concordance with measurements at certain wavelength range but with different curve tendencies. Differences in the reflectance response between regions are attributed to the limited size of the simulated structure and the BSi heterogeneity.

The second technique, consisting of modelling single equivalent structure shapes, shows improved simulated reflectances compared to measurements when the height distribution is similar to the global BSi height distribution (single cone or inverted cone shapes). In addition, improved concordance was obtained when the equivalent structure height is based on the global BSi height STD, compared with the height based on the global mean depth and the mean normal surface angle. This result suggests that by using the STD as structure height, it compensates the strong local variations of the mean height observed in the BSi topography.

In the last modelling technique, unit cells with 4 inverted cones are used in order to introduce variations of the structure height and diameter to break the periodicity of the single structure unit cell and therefore with greater resemblance to BSi topography. The results obtained consist in smoother reflectance responses with improved tendencies with respect to the ones observed in measurements, while the mean reflectance did not change due to the cells being designed with the same topographic parameters as the single structure unit cell. The multi-structure unit cell provides 8 degrees of freedom allowing to model unit cells having height distributions 
with greater similarity to the ones measured from BSi samples, which would imply further improvement in the reflectance matching.

The different multi-structure unit cell configurations $\left(1^{\text {st. }}\right.$ and $2^{\text {nd. }}$ cases $)$ provide about the same reflectance response with NRMSE with experimental measurements below $0.25 \%$ for wavelength range between 450 and $950 \mathrm{~nm}$. The remaining fluctuations observed in the reflectance of the multi-structure unit cell denote the trade-off between cell simplicity and reflectance accuracy.

The reduced average reflectance observed in simulations of multi-structure unit cells with respect to the single-structure cell at different AOI, suggests that surfaces having a disordered structure distribution with different dimensions such as BSi are preferable in cases where lower reflectance is required at different irradiation AOI than surfaces having perfectly organized structures with similar dimensions.

\section{References}

[1] Spinelli P, Verschuuren M and Polman A 2012 Nature communications 3692

[2] Kelzenberg M D, Boettcher S W, Petykiewicz J A, Turner-Evans D B, Putnam M C, Warren E L, Spurgeon J M, Briggs R M, Lewis N S and Atwater H A 2010 Nature materials 9 239-244

[3] Steglich M, Lehr D, Ratzsch S, Käsebier T, Schrempel F, Kley E B and Tünnermann A 2014 Laser 85 Photonics Reviews 8 L13-L17

[4] Dussart R, Mellhaoui X, Tillocher T, Lefaucheux P, Volatier M, Socquet-Clerc C, Brault P and Ranson P 2005 Journal of Physics D: Applied Physics 383395

[5] Lee C, Bae S Y, Mobasser S and Manohara H 2005 Nano letters 5 2438-2442

[6] Koynov S, Brandt M S and Stutzmann M 2006 Applied Physics Letters 88203107

[7] Her T-H, Finlay R J, Wu C, Deliwala S and Mazur E 1998 Applied Physics Letters 73 1673-75

[8] Nguyen K, Basset P, Marty F, Leprince-Wang Y and Bourouina T 2013 Journal of Applied Physics 113194903

[9] Oh J, Yuan H C and Branz H M 2012 Nature nanotechnology 7 743-748

[10] Carey J E, Crouch C H, Shen M and Mazur E 2005 Optics Letters 30 1773-1775

[11] Nguyen K, Abi-Saab D, Basset P, Richalot E, Malak M, Pavy N, Flourens F, Marty F, Angelescu D, Leprince-Wang Y and Bourouina T 2012 Microsystem Technologies 18 1807-1814

[12] Lehr D, Helgert M, Sundermann M, Morhard C, Pacholski C, Spatz J P and Brunner R 2010 Optics express 18 23878-23890

[13] Kroll M, Käsebier T, Otto M, Salzer R, Wehrspohn R, Kley E B, Tünnermann A and Pertsch T 2010 SPIE Photonics Europe (International Society for Optics and Photonics) pp 772505

[14] Zhu F Y, Wang Q Q, Zhang X S, Hu W, Zhao X and Zhang H X 2014 Nanotechnology 25 185705

[15] Abi Saab D, Trawick M, Basset P, Marty F and Angelescu D E 2014 Design, Test, Integration and Packaging of MEMS/MOEMS (DTIP), IEEE Symposium pp 347-350

[16] Palmer J M 1995 The measurement of transmission, absorption, emission, and reflection Handbook of Optics vol 2 (McGraw-Hill, New York)

[17] Garnett J M 1906 Philosophical Transactions of the Royal Society of London. Series A, Containing Papers of a Mathematical or Physical Character 237-288

[18] Anzai H, Saikawa M, Naito Y and Mizumoto T 1995 Electromagnetic Compatibility. IEEE International Symposium pp 563-567

[19] Hull R 1999 Properties of crystalline silicon (INSPEC) 